EGU2020-9190

https://doi.org/10.5194/egusphere-egu2020-9190

EGU General Assembly 2020

(c) Author(s) 2020. This work is distributed under

the Creative Commons Attribution 4.0 License.

\title{
BEL1D: 1D imaging using geophysical data in the framework of Bayesian Evidential Learning
}

\author{
Hadrien Michel ${ }^{1,2,3}$, Frédéric Nguyen ${ }^{1}$, and Thomas Hermans ${ }^{2}$ \\ ${ }^{1}$ University of Liège, Faculty of Applied Sciences, Urban and Environmental Engineering Department, Liège, Belgium \\ (hadrien.michel@uliege.be) \\ ${ }^{2}$ Ghent University, Faculty of Sciences, Department of Geology, Ghent, Belgium \\ ${ }^{3}$ F.R.S.-FNRS (Fonds de la Recherche Scientifique), Brussels, Belgium
}

BEL1D has been newly introduced to the community as a viable algorithm for the stochastic interpretation of geophysical data in the form of 1D geological models. It relies on a simplified version of the Bayesian problem in reduced space called Bayesian Evidential Learning. However, the method is closer to machine learning than classical McMC approaches since it can be separated into a learning process followed by a prediction part. The learning phase consists in constituting statistical relationships between models parameters and geophysical data from a training set of numerical models. The prediction phase then samples the previous relationships according to field data. Compared to other stochastic methods such as McMC, BEL1D as key advantages: 1) it converges easily as long as the prior is consistent with the unique input parameter being the size of the training set, 2) every model in the posterior is drawn independently, making it easy to trace back their origin, 3) the CPU times are similar to McMC, but the method can be fully parallelized and the learning process can be done before data acquisition, leading to quasi instantaneous prediction of the posterior. BEL1D already has led to successful applications on surface nuclear magnetic resonance data as well as dispersion curves from surface waves analysis. Nonetheless, the method is not limited to those two examples and can be implemented for any 1D geophysical method as long as a forward model is provided. Currently, the method is implemented for blocky imaging but will be extended to non-blocky models in the future. The open-source codes are readily available.

How to cite: Michel, H., Nguyen, F., and Hermans, T.: BEL1D: 1D imaging using geophysical data in the framework of Bayesian Evidential Learning, EGU General Assembly 2020, Online, 4-8 May 2020, EGU2020-9190, https://doi.org/10.5194/egusphere-egu2020-9190, 2020 